\title{
SMOOTH DYNAMICS ON WEIERSTRASS NOWHERE DIFFERENTIABLE CURVES
}

\author{
BRIAN R. HUNT AND JAMES A. YORKE
}

\begin{abstract}
We consider a family of smooth maps on an infinite cylinder which have invariant curves that are nowhere smooth. Most points on such a curve are buried deep within its spiked structure, and the outermost exposed points of the curve constitute an invariant subset which we call the "facade" of the curve. We find that for surprisingly many of the maps in the family, all points in the facades of their invariant curves are eventually periodic.
\end{abstract}

\section{INTRODUCTION}

In the study of chaotic attractors of dynamical systems, one mechanism by which these attractors have been observed to be destroyed as system parameters vary is the boundary crisis $[3,4]$. In a boundary crisis, the attractor collides with an unstable periodic orbit on the boundary of its basin of attraction (the set of initial conditions whose trajectories approach the attractor as time increases) as some parameter reaches a critical value. The basin boundary is, like the attractor, invariant under the dynamical system, and is fractal in many cases $[1,2,5-7,9,11-16]$. That a collision between these invariant sets should tend to involve periodic orbits $[2-6,12]$ may reflect a tendency toward periodicity among the most exposed points of these sets. In this paper we find such a tendency for a family of two-dimensional maps which have basin boundaries that are fractal curves. A similar phenomenon is observed for the Hénon map in [2].

In [6], the map

$$
\begin{gathered}
\theta_{n+1}=2 \theta_{n} \quad(\bmod 1), \\
z_{n+1}=\alpha z_{n}+z_{n}^{2}+\beta \cos 2 \pi \theta_{n}
\end{gathered}
$$

(where $\theta$ lies in $[0,1)$ and $z$ is real) is found, for appropriate values of $\alpha$ and $\beta$, to have a chaotic attractor whose basin of attraction has a fractal boundary. As $\alpha$ is increased past a critical value, an unstable fixed point on the attractor

Received by the editors April 10, 1989. Presented to the SIAM Conference on Dynamical Systems in Orlando, Florida, May 10, 1990.

1980 Mathematics Subject Classification (1985 Revision). Primary 26A27; Secondary 42A55.

The second author's research was partially supported by a grant from AFOSR. 
collides with an unstable fixed point on the basin boundary and the attractor is destroyed. In this paper we study the family of maps

$$
\begin{gathered}
\theta_{n+1}=2 \theta_{n} \quad(\bmod 1), \\
z_{n+1}=\mu\left(z_{n}-f\left(\theta_{n}\right)\right),
\end{gathered}
$$

where $1<\mu<2$ and $f$ is $C^{1}$ and periodic (with period one). When $f$ is a multiple of $\cos 2 \pi \theta,(1.1)$ can be thought of as a linearization of the previous map for values of $z$ near the basin boundary; this map has also been studied in $[6,11]$. It will later be shown that all initial conditions $\left(\theta_{0}, z_{0}\right)$ except those on the curve

$$
z=\sum_{n=0}^{\infty} \mu^{-n} f\left(2^{n} \theta\right)
$$

have unbounded trajectories; this curve is invariant under (1.1) and forms the boundary between those initial conditions whose trajectories have $z_{n} \rightarrow+\infty$ as $n \rightarrow \infty$ and those for which $z_{n} \rightarrow-\infty$ as $n \rightarrow \infty$. While this curve is continuous, it does not in general retain any smoothness that $f$ might have, as is indicated by the formal expansion for $d z / d \theta$,

$$
\sum_{n=0}^{\infty}\left(\frac{2}{\mu}\right)^{n} f^{\prime}\left(2^{n} \theta\right),
$$

which in general diverges since $\mu<2$. In fact, such curves generally have dimension greater than one [10]. The case $f(\theta)=\cos 2 \pi \theta$ is of particular historical interest, since then (1.2) is the equation of the Weierstrass nowhere differentiable curve (see, for example, [8]).

Let $g$ be a continuous function. We say that a point $(\varphi, g(\varphi))$ is a "point on the facade" of the curve $z=g(\theta)$ if there exists a $C^{1}$ function $h$ defined in a neighborhood of $\varphi$ which is equal to $g$ at $\varphi$ and whose graph does not cross that of $g$ (that is, either $h \geq g$ on the entire domain of $h$, or $h \leq g$ throughout the domain). We refer to such a function $h$ as a "probe" for $g$ at $\varphi$. We call the set of points at which $g$ has a probe from above the "upper facade" of the graph of $g$, and define the "lower facade" analogously.

In $\S 2$, we derive (1.2), and discuss the dynamics of (1.1) on this curve. We then examine the facade of this curve in the special case $f(\theta)=\cos 2 \pi \theta$. Next we obtain some results concerning the invariance of the facade under (1.1), and use them to show that most nonperiodic points on the invariant curve can never be on the facade unless the curve is $C^{1}$, which we in turn show is only the case for a relatively small set of functions $f$. In $\S 3$ we show that any point on the facade which is not eventually periodic can be removed from the facade by an arbitrarily small $C^{1}$ perturbation of $f$, whereas for any given periodic orbit there is an open (in the $C^{1}$ topology) set of $f$ for which the facade consists of that periodic orbit together with its stable manifold. 


\section{Preliminaries}

We now describe the compact invariant sets of (1.1). Let us think of $\theta$ as a parameter of a circle $T$ with unit circumference. Since $f$ is then a continuous function on the compact set $T$, it must be bounded; say $|f(\theta)| \leq M$ for all $\theta \in T$. Letting $z_{*}=\mu M /(\mu-1)$ we rewrite $(1.1 \mathrm{~b})$ as

$$
\begin{aligned}
z_{n+1}-z_{*} & =\mu\left(z_{n}-f\left(\theta_{n}\right)\right)-\mu M /(\mu-1) \\
& =\mu\left(z_{n}-z_{*}\right)+\mu\left(M-f\left(\theta_{n}\right)\right) \geq \mu\left(z_{n}-z_{*}\right) .
\end{aligned}
$$

Thus if $z_{k}>z_{*}$ for some $k \geq 0$, then $z_{n} \rightarrow \infty$ as $n \rightarrow \infty$. Let $A^{+}$be the set of $(\theta, z)$ with $z>z_{*}$ and $B^{+}$be the set of initial conditions for which $z_{n} \rightarrow \infty$ as $n \rightarrow \infty$; then $A^{+} \subset B^{+}$. Furthermore, $B^{+}$is open, for if $\left(\theta_{0}, z_{0}\right) \in B^{+}$, its trajectory must eventually enter $A^{+}$, which is open, and therefore initial conditions in a neighborhood of $\left(\theta_{0}, z_{0}\right)$ have their trajectories enter $A^{+}$at the same time, so as with all trajectories that enter $A^{+}$they have $z_{n} \rightarrow \infty$ as $n \rightarrow \infty$. Similarly, the set $B^{-}$of initial conditions for which $z_{n} \rightarrow-\infty$ as $n \rightarrow \infty$ is open and contains the set $A^{-}$of $(\theta, z)$ with $z<-z_{*}$. Then any vertical line $\theta=$ constant must intersect both $B^{+}$and $B^{-}$, but such a line must also contain at least one point that lies in neither set, since both sets are open.

Now observe that in (1.1), the dynamics of $\theta$ are independent of $z$, while for a given $\theta$ value, $z$ is transformed by a linear map with slope $\mu$. In other words, any two trajectories with the same initial $\theta_{0}$ have the same $\theta$-coordinate for all $n$, while their $z$-coordinates grow apart geometrically. Thus in fact only one point on any given vertical line lies outside both $B^{+}$and $B^{-}$; for a given $\theta$ let $g(\theta)$ be the $z$-coordinate of that point. The graph $G$ of $z=g(\theta)$ consists of all initial conditions whose trajectories remain bounded for all $n$, and hence must be invariant under (1.1). Furthermore, any compact invariant set must be a subset of $G$.

The observation that vertical lines are mapped linearly to vertical lines, stretched by a factor of $\mu$, also leads to a derivation of the formula for $g(\theta)$ contained in (1.2). Since points are repelled by $G$ under our map, they must be attracted to it under the inverse process. Though the inverse of $(1.1)$ is doublevalued, given a trajectory $\theta_{0}, \theta_{1}, \ldots$ of (1.1a) ending up at some $\theta_{N}$, and a value of $z_{N}$, we can iterate (1.1b) backwards $N$ times to obtain

$$
z_{0}=\mu^{-N} z_{N}+\sum_{n=0}^{N-1} \mu^{-n} f\left(\theta_{n}\right)
$$

To find $g(\theta)$ for a given $\theta$, we let $\theta_{0}=\theta$; then $\theta_{n}=2^{n} \theta$ for all $n \geq 0$. (Multiplication of elements of $T$ by integers is of course done modulo one.) Letting $N \rightarrow \infty$ while keeping our choice for $z_{N}$ constant, we end up with the 
formula

$$
g(\theta)=\sum_{n=0}^{\infty} \mu^{-n} f\left(2^{n} \theta\right) .
$$

Now consider for a moment the dynamics of (1.1) on the invariant set $G$ in light of the fact that $G$ is the graph of a function of $\theta$. The point $(\varphi, g(\varphi)) \in G$ must be mapped to a point in $G$ with $\theta$-coordinate $2 \varphi$; the only such point is $(2 \varphi, g(2 \varphi))$. Thus given the $1-1$ correspondence $(\theta, g(\theta)) \Leftrightarrow \theta$ between $G$ and $T$, the dynamics of (1.1) on $G$ are equivalent to the dynamics of (1.1a) on $T$. This correspondence allows us to characterize the periodic and eventually periodic points in $G$. A periodic point must have $2^{m} \theta=\theta$ for some positive integer $m$, whence $\left(2^{m}-1\right) \theta=0$, and $\theta$ must be a rational number with denominator dividing $2^{m}-1$; in particular the denominator must be odd. On the other hand, for any odd positive integer $q$, the map $\theta \mapsto 2 \theta$ is $1-1$ from the set $\{p / q \mid 0 \leq p \leq q-1\}$ to itself, and thus each point in this set is periodic. Therefore, the periodic points of $G$ are precisely those whose $\theta$-coordinates are rational numbers with odd denominator. The eventually periodic points are those which are mapped to periodic points in finite time, that is, those for which $2^{m} \theta$ is periodic for some positive integer $m$. The set of such points is then simply the set of all rational numbers in $[0,1)$. It is therefore our aim to show that for most functions $f \in C^{1}(T)$ the facade of $G$ consists only of points whose $\theta$-coordinates are rational.

Next let us investigate the facade of $G$ in the special case $f(\theta)=\cos 2 \pi \theta$. First consider the upper facade. One point that must be on it is the point at which $g$ takes on its maximum, which in this case is the fixed point $\theta=0$, since $\cos \left(2 \pi 2^{n} \theta\right)$ takes on its maximum there for all $n$. As we will now see, from just this one point we can generate an infinite set of points in the upper facade. Because of the invariance of $G$ (or directly from (2.1)),

$$
g(2 \theta)=\mu(g(\theta)-f(\theta)) .
$$

It follows that if a point is on the upper facade of $G$, so are both of its preimages. (If $h$ is a probe for $g$ at $\varphi$, then $\mu^{-1} h(2 \theta)+f(\theta)$, defined in an appropriate neighborhood of one of the preimages of $\varphi$, is a probe for $g$ at that preimage.) Thus by induction all possible backward iterates of $(0, g(0))$ are on the upper facade of $G$, that is, all $(\theta, g(\theta))$ for which $\theta$ is a diadic rational $\left(\theta=k / 2^{m}\right.$ for nonnegative integers $k$ and $\left.m, k<2^{m}\right)$. In fact, these are the only points in the facade, as we will now prove.

2.1. Proposition. If $f$ is sufficiently close to $\cos 2 \pi \theta$ in $C^{1}(T)$, then the upper facade of $G$ is the set $D$ of $(\theta, g(\theta))$ with $\theta$ a diadic rational.

Proof. It suffices to show that all points not in $D$ are not on the upper facade. Then the maximum of $g$ will have to occur at a point of $D$; this point must be on the upper facade. As we have mentioned, (2.2) implies that the preimages of a point on the upper facade are also in the upper facade, and similarly the reverse is true: the image under (1.1) of a point on the upper facade is also on 
the upper facade. Since all points in $D$ are eventually mapped to $(0, g(0))$, it will follow that the upper facade contains $(0, g(0))$, and thus as above all points in $D$ will be on the upper facade.

Let us define the asymmetric difference quotient

$$
\Delta g(\theta, \varphi)=\frac{g(\theta)-g(\varphi)}{|\theta-\varphi|},
$$

where $|\theta-\varphi|$ means distance along the circle $T$, e.g., $|.9-.1|=.2$. If $\varphi \in T$ is not a diadic rational, then for all integers $m \geq 0$ there is an integer $k$ with $0 \leq k<2^{m}$ such that $k / 2^{m}<\varphi<(k+1) / 2^{m}$. We will show that there exists a positive constant $C$ independent of $m$ and $\varphi$ for which

$$
\max \left(\Delta g\left(\frac{k}{2^{m}}, \varphi\right), \Delta g\left(\frac{k+1}{2^{m}}, \varphi\right)\right) \geq C\left(\frac{2}{\mu}\right)^{m} .
$$

Then if $h$ were a probe for $g$ at $\varphi,(2.3)$ would hold with $g$ replaced by $h$, and since $C(2 / \mu)^{m} \rightarrow \infty$ as $m \rightarrow \infty$, this would contradict the requirement that $h$ be differentiable at $\varphi$. Thus (2.3) implies that any $(\varphi, g(\varphi))$ not in $D$ is not in the facade.

We wish to prove $(2.3)$ for all $\varphi \in\left(k / 2^{m},(k+1) / 2^{m}\right)$. We first establish it when $\varphi$ is the midpoint of the interval, then by induction extend it to all diadic rationals in the interval, and finally by continuity extend it to the whole interval. Now if $f(\theta)$ is within $\varepsilon$ of $\cos 2 \pi \theta$ in $C^{1}(T)$, then

$$
\begin{aligned}
\max & \left(\Delta g\left(\frac{k}{2^{m}}, \frac{2 k+1}{2^{m+1}}\right), \Delta g\left(\frac{k+1}{2^{m}}, \frac{2 k+1}{2^{m+1}}\right)\right) \\
& \geq \frac{1}{2}\left(\Delta g\left(\frac{k}{2^{m}}, \frac{2 k+1}{2^{m+1}}\right)+\Delta g\left(\frac{k+1}{2^{m}}, \frac{2 k+1}{2^{m+1}}\right)\right) \\
& =2^{m}\left(g\left(\frac{k}{2^{m}}\right)+g\left(\frac{k+1}{2^{m}}\right)-2 g\left(\frac{2 k+1}{2^{m+1}}\right)\right) \\
& =2^{m} \sum_{n=0}^{\infty} \mu^{-n}\left(f\left(2^{n} \frac{k}{2^{m}}\right)+f\left(2^{n} \frac{k+1}{2^{m}}\right)-2 f\left(2^{n} \frac{2 k+1}{2^{m+1}}\right)\right) \\
& \geq 2^{m} \sum_{n=0}^{m} \mu^{-n}\left(\cos \frac{2 \pi k}{2^{m-n}}+\cos \frac{2 \pi(k+1)}{2^{m-n}}-2 \cos \frac{2 \pi(2 k+1)}{2^{m-n+1}}-2 \varepsilon \frac{1}{2^{m-n+1}}\right) \\
& =2^{m} \sum_{n=0}^{m} \mu^{n-m}\left(\cos \frac{2 \pi k}{2^{n}}+\cos \frac{2 \pi(k+1)}{2^{n}}-2 \cos \frac{2 \pi(2 k+1)}{2^{n+1}}\right)-\varepsilon \sum_{n=0}^{m}\left(\frac{2}{\mu}\right)^{n} \\
& \geq\left(\frac{2}{\mu}\right)^{m} \sum_{n=0}^{m} \mu^{n}\left(\cos 0+\cos \frac{2 \pi}{2^{n}}-2 \cos \frac{2 \pi}{2^{n+1}}\right)-\varepsilon \frac{(2 / \mu)^{m}}{1-\mu / 2} \\
& =\left(\frac{2}{\mu}\right)^{m}\left(\sum_{n=0}^{m} \mu^{n}\left(1-(2-\mu) \cos \frac{2 \pi}{2^{n+1}}\right)+1-\mu^{m+1} \cos \frac{2 \pi}{2^{m+1}}-\frac{2}{2-\mu} \varepsilon\right) \\
& \geq\left(\frac{2}{\mu}\right)^{m}\left(3-\mu+\sum_{n=1}^{m} \mu^{n}(\mu-1)+1-\mu^{m+1}-\frac{2}{2-\mu} \varepsilon\right)^{m}
\end{aligned}
$$


(continued)

$$
\begin{aligned}
& =\left(\frac{2}{\mu}\right)^{m}\left(4-\mu+\mu^{m+1}-\mu-\mu^{m+1}-\frac{2}{2-\mu} \varepsilon\right) \\
& =\left(\frac{2}{\mu}\right)^{m}\left(2(2-\mu)-\frac{2}{2-\mu} \varepsilon\right) .
\end{aligned}
$$

Thus, if $\varepsilon<(2-\mu)^{2}$ then (2.3) holds for $\varphi=(2 k+1) / 2^{m+1}$ with

$$
C=2(2-\mu)-\frac{2}{2-\mu} \varepsilon>0 .
$$

Next we employ the following transitivity property for asymmetric difference quotients. For any $\theta, \varphi, \psi \in T$,

$$
\begin{aligned}
\Delta g(\theta, \varphi) & =\frac{g(\theta)-g(\psi)+g(\psi)-g(\varphi)}{|\theta-\varphi|} \\
& =\frac{|\theta-\psi| \Delta g(\theta, \psi)+|\psi-\varphi| \Delta g(\psi, \varphi)}{|\theta-\varphi|} \\
& \geq \frac{|\theta-\psi| \Delta g(\theta, \psi)+|\psi-\varphi| \Delta g(\psi, \varphi)}{|\theta-\psi|+|\psi-\varphi|} \\
& \geq \min (\Delta g(\theta, \psi), \Delta g(\psi, \varphi)),
\end{aligned}
$$

provided the latter two difference quotients are positive. Now let $\varphi$ be midway between $k / 2^{m}$ and $(2 k+1) / 2^{m+1}$. By our previous estimate (with $k$ replaced by $2 k$ and $m$ by $m+1)$,

$$
\max \left(\Delta g\left(\frac{k}{2^{m}}, \varphi\right), \Delta g\left(\frac{2 k+1}{2^{m+1}}, \varphi\right)\right) \geq C\left(\frac{2}{\mu}\right)^{m+1}>C\left(\frac{2}{\mu}\right)^{m} .
$$

If the former difference quotient is larger, (2.3) follows immediately; if the latter is larger, then, by our transitivity property,

and

$$
\begin{aligned}
\Delta g\left(\frac{k}{2^{m}}, \varphi\right) & \geq \min \left(\Delta g\left(\frac{k}{2^{m}}, \frac{2 k+1}{2^{m+1}}\right), \Delta g\left(\frac{2 k+1}{2^{m+1}}, \varphi\right)\right) \\
& \geq \min \left(\Delta g\left(\frac{k}{2^{m}}, \frac{2 k+1}{2^{m+1}}\right), C\left(\frac{2}{\mu}\right)^{m}\right)
\end{aligned}
$$

$$
\begin{aligned}
\Delta g\left(\frac{k+1}{2^{m}}, \varphi\right) & \geq \min \left(\Delta g\left(\frac{k+1}{2^{m}}, \frac{2 k+1}{2^{m+1}}\right), \Delta g\left(\frac{2 k+1}{2^{m+1}}, \varphi\right)\right) \\
& \geq \min \left(\Delta g\left(\frac{k+1}{2^{m}}, \frac{2 k+1}{2^{m+1}}\right), C\left(\frac{2}{\mu}\right)^{m}\right),
\end{aligned}
$$

in each case provided that the difference quotient on the right is positive. But by our previous estimate, at least one of the two right-hand difference quotients must exceed $C(2 / \mu)^{m}$, and thus $(2.3)$ holds for the new value of $\varphi$. Similarly, (2.3) holds for $\varphi$ midway between $(2 k+1) / 2^{m+1}$ and $(k+1) / 2^{m}$; applied inductively, this argument extends $(2.3)$ to all diadic rationals between $k / 2^{m}$ and $(k+1) / 2^{m}$. Since the expression on the left of $(2.3)$ is continuous for $\varphi \in\left(k / 2^{m},(k+1) / 2^{m}\right)$, the inequality must then hold for all such $\varphi$, and the proof is complete. 
The lower facade of $G$ is not found as easily. We first claim that $g$ is minimized at both points in the period two orbit $\{1 / 3,2 / 3\}$. At these values of $\theta, \cos \left(2 \pi 2^{n} \theta\right)=-1 / 2$ for all $n$, so for any $\theta$,

$$
g(\theta)-g\left(\frac{1}{3}\right)=g(\theta)-g\left(\frac{2}{3}\right)=\sum_{n=0}^{\infty} \mu^{-n}\left(\cos \left(2 \pi 2^{n} \theta\right)+\frac{1}{2}\right) .
$$

To show that this sum is nonnegative, we show that any negative term is succeeded by a positive term with greater absolute value. In other words, we show that if $\cos 2 \pi \theta+1 / 2$ is negative,

$$
\mu^{-1}\left(\cos 4 \pi \theta+\frac{1}{2}\right)>-\left(\cos 2 \pi \theta+\frac{1}{2}\right) .
$$

Now if $\cos 2 \pi \theta+1 / 2$ is negative, $1 / 3<\theta<2 / 3$; we assume $1 / 3<\theta<1 / 2$, the other case being covered by symmetry. At $\theta=1 / 3$, the above quantities are equal, so the inequality will follow from the corresponding inequality between the derivatives of each side, which is $-2 \mu^{-1} \sin 4 \pi \theta>\sin 2 \pi \theta$, or equivalently $2 \mu^{-1} \sin 4 \pi(1 / 2-\theta)>\sin 2 \pi(1 / 2-\theta)$. Since $2 \mu^{-1}>1$, this is indeed true for $0<1 / 2-\theta<1 / 6$, that is for $1 / 3<\theta<1 / 2$. Therefore $g$ is minimized at $1 / 3$ and $2 / 3$ as claimed. As above, it follows that all backward iterates of these points are on the lower facade of $G$, the backward iterates being those $(\theta, g(\theta))$ for which $\theta=k /\left(3 \cdot 2^{m}\right)$ for nonnegative integers $k$ and $m$ with $k$ not a multiple of 3 and $k<3 \cdot 2^{m}$. And at least for some values of $\mu$ (e.g., $\mu \leq 1.5$ ), it can be shown that these are the only points on the lower facade, and that this property persists for $f$ sufficiently close to $\cos 2 \pi \theta$ in $C^{1}(T)$.

Returning to the case of general $f \in C^{1}(T)$, let us start writing $\lambda$ for $\mu^{-1}$; then $1 / 2<\lambda<1$. Also, let us think of (2.1) as defining a linear operator $W$ from $C^{1}(T)$ to $C(T)$, writing $W f$ instead of $g$ from now on. In this notation, (2.1) becomes

$$
W f(\theta)=\sum_{n=0}^{\infty} \lambda^{n} f\left(2^{n} \theta\right),
$$

and the analogue of $(2.2)$ is

$$
\lambda W f(2 \theta)=W f(\theta)-f(\theta) .
$$

Finally, we define the facade of the function $W f$ to be the set of $\theta \in T$ for which $(\theta, W f(\theta))$ is on the facade of its graph.

Now as in the cosine example, (2.5) implies that a point $\varphi$ is on the upper facade of $W f$ if and only if $2 \varphi$ is, for (2.5) describes a local diffeomorphism which maps the graph of $W f$ near $\varphi$ to its graph near $2 \varphi$, and under which a probe at one point is transformed into a probe at the other point. (The same is of course true for the lower facade; all future results, though only stated for the upper facade, will apply to the lower facade, too). The following proposition extends the above observations. 
2.2. Proposition. Given $f \in C^{1}(T)$ and a point $\varphi$ on the upper facade of $W f$, the following points are also on the upper facade: all backward and forward iterates of $\varphi$, and all limit points of the forward trajectory starting at $\varphi$.

Proof. Since the upper facade is closed under (1.1) and its (double-valued) inverse, all backward and forward iterates of $\varphi$ are on the upper facade. Let $h_{0}(\theta)$ be a probe for $W f$ at $\varphi$, and define probes $h_{1}, h_{2}, h_{3}, \ldots$ at $2 \varphi, 4 \varphi, 8 \varphi, \ldots$ by

$$
h_{n+1}(2 \theta)=\lambda^{-1}\left(h_{n}(\theta)-f(\theta)\right), \quad\left|\theta-2^{n} \varphi\right|<\frac{1}{4},
$$

for $n \geq 0$. Notice that the domains of these probes will double in size at each step until for some $n$ the radius of the neighborhood of $2^{n} \varphi$ on which $h_{n}$ is defined exceeds $1 / 4$; then all future $h_{n}$ will be defined on an open neighborhood of $2^{n} \varphi$ with radius $1 / 2$, that is all but one point of $T$. We wish to show that the sequences $\left\{h_{n}\right\}$ and $\left\{h_{n}^{\prime}\right\}$ are uniformly bounded and equicontinuous. Then for any limit point $\psi=\lim _{k \rightarrow \infty} 2^{n_{k}} \varphi$ of the forward trajectory of $\varphi$, the probes $\left\{h_{n_{k}}\right\}$ will eventually be defined on a common neighborhood of $\psi$, so by the Ascoli-Arzela theorem some subsequence of $\left\{h_{n_{k}}\right\}$ will converge to a $C^{1}$ function $h$ defined near $\psi$. It is not hard to show that such an $h$ must be a probe for $W f$ at $\psi$.

We first show that the sequence $\left\{h_{n}^{\prime}\right\}$ is uniformly bounded; this implies not only that $\left\{h_{n}\right\}$ is equicontinuous, but also, since $h_{n}\left(2^{n} \varphi\right)=W f\left(2^{n} \varphi\right)$ for all $n$ and $W f$ is bounded, that $\left\{h_{n}\right\}$ is uniformly bounded. Let $M$ be the maximum value of $\left|f^{\prime}\right|$ on $T$, and for all $n$ let $M_{n}$ be the maximum value of $\left|h_{n}^{\prime}\right|$. Differentiating (2.6) and dividing by 2 yields

$$
h_{n+1}^{\prime}(2 \theta)=(2 \lambda)^{-1}\left(h_{n}^{\prime}(\theta)-f^{\prime}(\theta)\right) \text {, }
$$

and thus

$$
M_{n+1} \leq(2 \lambda)^{-1}\left(M_{n}+M\right) .
$$

Since $2 \lambda>1$, the above inequality insures that $\left\{M_{n}\right\}$ remains bounded as $n \rightarrow \infty$; specifically we have

$$
\limsup _{n \rightarrow \infty} M_{n} \leq \frac{M}{2 \lambda-1} .
$$

We now need only show that $\left\{h_{n}^{\prime}\right\}$ is equicontinuous. Choose $\varepsilon>0$. Since $f^{\prime}$ and $h_{0}^{\prime}$ are continuous on a compact set, there exists a $\delta>0$ such that $\left|f^{\prime}(\theta)-f^{\prime}(\psi)\right|<(2 \lambda-1) \varepsilon$ and $\left|h_{0}^{\prime}(\theta)-h_{0}^{\prime}(\psi)\right|<\varepsilon$ whenever $|\theta-\psi|<\delta$. Suppose now that for some $n \geq 0,\left|h_{n}^{\prime}(\theta)-h_{n}^{\prime}(\psi)\right|<\varepsilon$ whenever $|\theta-\psi|<\delta$. Then by (2.7), if $|2 \theta-2 \psi|<\delta$ (and $|\theta-\psi|<\delta / 2$ ), then

$$
\begin{aligned}
\left|h_{n+1}^{\prime}(2 \theta)-h_{n+1}^{\prime}(2 \psi)\right| & \leq(2 \lambda)^{-1}\left(\left|h_{n}^{\prime}(\theta)-h_{n}^{\prime}(\psi)\right|+\left|f^{\prime}(\theta)-f^{\prime}(\psi)\right|\right) \\
& <(2 \lambda)^{-1}(\varepsilon+(2 \lambda-1) \varepsilon)=\varepsilon .
\end{aligned}
$$

Therefore, by induction $\left\{h_{n}^{\prime}\right\}$ is equicontinuous, and the proof is complete. 
Though the preceding proposition may seem geared toward showing the facade has many points, it will also be helpful in excluding certain points. For example, since $\theta \mapsto 2 \theta(\bmod 1)$ is ergodic, almost every initial point in $T$ has a dense trajectory. Consider the consequences of having such a point in the facade of $W f$. Since every point in $T$ will be a limit point for its trajectory, Proposition 2.2 implies that every point must be in the facade, which means $W f$ is smoother than we would normally expect. In fact, we will be able to prove that $W f$ must be $C^{1}$ in this situation, with the help of the following lemma.

2.3. Lemma. Let $\varphi$ be a point on the upper facade of $W f$, and let $M$ be the maximum value of $\left|f^{\prime}\right|$ on $T$. Then if $\psi$ is either a limit point of the forward trajectory of $\varphi$ or a member $2^{n} \varphi$ of this trajectory for $n$ sufficiently large, then

$$
W f(\theta)-W f(\psi) \leq \frac{M+1}{2 \lambda-1}|\theta-\psi| \quad \forall \theta \in T .
$$

Proof. Let $h_{0}(\theta)$ be a probe for $W f$ at $\varphi$, and define $h_{n}(\theta)$ and $M_{n}$ for $n \geq 0$ as in the previous proof. Let $L=(M+1) /(2 \lambda-1)$. By $(2.8), M_{n} \leq L$ for $n$ sufficiently large; then for all $\theta$ in the domain of $h_{n}$,

$$
W f(\theta)-W f\left(2^{n} \varphi\right)=W f(\theta)-h_{n}\left(2^{n} \varphi\right) \leq h_{n}(\theta)-h_{n}\left(2^{n} \varphi\right) \leq L\left|\theta-2^{n} \varphi\right| \text {. }
$$

Since $W f$ is continuous and the domain of $h_{n}$ extends to all but one point of $T$ for $n$ sufficiently large, the above inequality holds for all $\theta \in T$ when $n$ is sufficiently large. Then for any limit point $\psi=\lim _{k \rightarrow \infty} 2^{n_{k}} \varphi$ and all $\theta \in T$,

$$
\begin{aligned}
W f(\theta)-W f(\psi) & =\lim _{k \rightarrow \infty}\left(W f(\theta)-W f\left(2^{n_{k}} \varphi\right)\right) \\
& \leq \lim _{k \rightarrow \infty} L\left|\theta-2^{n_{k}} \varphi\right|=L|\theta-\psi| .
\end{aligned}
$$

2.4. Proposition. If the upper facade of $W f$ contains a point with a dense trajectory, then $W f$ is $C^{1}$.

Proof. We note for future reference the two steps in our proof: first we show that $W f$ must be Lipschitz with constant $(M+1) /(2 \lambda-1)$, where $M$ is the maximum value of $\left|f^{\prime}\right|$, then we show that whenever $W f$ is Lipschitz it must be $C^{1}$. The first step follows from the preceding lemma: since all $\psi \in T$ are limit points of the forward trajectory of $\varphi,(2.9)$ holds for all $\theta, \psi \in T$, which means that $W f$ is Lipschitz with the desired constant. Now suppose $W f$ is Lipschitz with any constant $L$. Then $W f$ has bounded variation, and therefore is differentiable almost everywhere. Because of the Lipschitz condition, $W f^{\prime}$ must lie in $L^{\infty}(T)$ with norm at most $L$. To prove that it is continuous we differentiate $(2.5)$ and divide by $2 \lambda$ to get

$$
W f^{\prime}(2 \theta)=(2 \lambda)^{-1}\left(W f^{\prime}(\theta)-f^{\prime}(\theta)\right) \text {. }
$$

Iterating $N$ times yields

$$
W f^{\prime}\left(2^{N} \theta\right)=(2 \lambda)^{-N} W f^{\prime}(\theta)-\sum_{n=0}^{N-1}(2 \lambda)^{n-N} f^{\prime}\left(2^{n} \theta\right) \text {. }
$$


Since $f$ is $C^{1}$, the above equation implies that in any neighborhood of $2^{N} \theta$, $W f^{\prime}$ is within $(2 \lambda)^{-N} L$ of a continuous function. Fixing $\varphi=2^{N} \theta$ and letting $N \rightarrow \infty$ implies that $W f^{\prime}$ is locally the uniform limit of continuous functions, and therefore is continuous at $\varphi$. Since $\varphi$ is arbitrary, $W f$ is indeed a $C^{1}$ function.

Given a subset $U$ of $T$, we define $S(U)$ to be the set of $f \in C^{1}(T)$ for which the upper facade of $W f$ contains $U$. As we have observed, Proposition 2.2 implies that if $\varphi$ has a dense trajectory, $S(\{\varphi\})=S(T)$. Proposition 2.4 then characterizes $S(T)$ as the set of $f \in C^{1}(T)$ for which $W f$ is $C^{1}$. The following proposition shows that $S(T)$ is a relatively small subset of $C^{1}(T)$.

2.5. Proposition. $S(T)$ is a proper closed (linear) subspace of $C^{1}(T)$.

Proof. Recall that $W$ is a linear operator from $C^{1}(T)$ to $C(T)$; since $C^{1}(T)$ is a subspace of $C(T)$, its inverse image under $W, S(T)$, is a subspace of $C^{1}(T)$. To see that this subspace is proper, we observe that Proposition 2.1 implies that $\cos 2 \pi \theta$ and nearby functions are not in $S(T)$, but $S(T)$ is not empty because it must contain every function of the form $f(\theta)=g(\theta)-\lambda g(2 \theta)$ with $g \in C^{1}(T)$, (for then $W f=g$ by (2.4)). Finally, to prove that $S(T)$ is closed, consider a sequence $\left\{f_{n}\right\}$ of functions in $S(T)$ which converge to some $f \in C^{1}(T)$. Notice from (2.4) that $W$ is bounded as well as linear: for all $g \in C^{1}(T)$,

$$
\max _{\theta \in T}|W g(\theta)| \leq \frac{1}{1-\lambda} \max _{\theta \in T}|g(\theta)| .
$$

It follows that $W f_{n} \rightarrow W f$ in $C(T)$ as $n \rightarrow \infty$; we wish to show that $W f$ is $C^{1}$. Since $\left\{f_{n}\right\}$ converges in the $C^{1}$ norm, there must be a uniform upper bound $M$ on $\left|f_{n}^{\prime}\right|$ for all $n$. Since each $W f_{n}$ belongs to $S(T)$, as noted in the proof of Proposition 2.4 each $W f_{n}$ is Lipschitz with (uniform) constant $(M+1) /(2 \lambda-1)$. Being the limit of $\left\{W f_{n}\right\}, W f$ must also be Lipschitz with the same constant, and thus by the proof of Proposition 2.4, $W f$ is $C^{1}$. Therefore $S(T)$ is closed, and the proof is complete.

These results imply that having $W f$ smooth is an exceptional case; since points in $T$ which are eventually periodic (that is, rational) do not have dense trajectories, they would now seem more likely than most points to be in the facade. However, there are many irrational points in $T$ which do not have dense trajectories either. For example, consider the set $Q$ of points in $T$ whose binary expansions do not contain two consecutive ones, which is a Cantor set and is invariant under $\theta \mapsto 2 \theta(\bmod 1)$. The forward trajectory of any point in $Q$ confines itself to $Q$, and is therefore not dense. In the following section we will show that such points are still unlikely to occur in the facade. 


\section{MAIN RESUlts}

One of the implications of Proposition 2.5 is that $S(T)$ has no interior. In other words, arbitrarily small $C^{1}$ perturbations of any $f$ in this set can eliminate all points with dense trajectories from the facade. We can say the same thing about $S(Q)$ for any invariant Cantor set $Q$, or in fact for any set $Q$ which contains an irrational number.

3.1. Theorem. If $Q \subset T$ contains any irrational number, then $S(Q)$ has no interior.

Proof. Let $\varphi$ be an irrational member of $Q$; it suffices to show that if $f \in$ $S(Q)$, there exist arbitrarily small perturbations of $f$ for which $\varphi$ is not on the facade of the perturbed $W f$. Let $\psi$ be a limit point of the forward trajectory of $\varphi$, and let $M$ be the maximum value of $\left|f^{\prime}\right|$ on $T$. Choose $\varepsilon>0$; we will construct a $C^{1}$ function $\tilde{f}$ for which $|\tilde{f}|$ and $\left|\tilde{f}^{\prime}\right|$ do not exceed $\varepsilon$, but for some $\theta \in T$

$$
W(f+\tilde{f})(\theta)-W(f+\tilde{f})(\psi)>\frac{M+\varepsilon+1}{2 \lambda-1}|\theta-\psi| .
$$

That is, (2.9) will not hold for $W(f+\tilde{f})$, and therefore by Lemma 2.3, $\varphi$ cannot be on the facade of $W(f+\tilde{f})$.

Now by Lemma 2.3, we can pick a positive integer $N$ such that if $n \geq N$,

$$
W f(\theta)-W f\left(2^{n} \varphi\right) \leq \frac{M+1}{2 \lambda-1}\left|\theta-2^{n} \varphi\right|
$$

for all $\theta \in T$. Since $\lambda<1<2 \lambda$, we may choose positive integers $l$ and $m$ such that

$$
(2 \lambda)^{l}\left(1-\frac{\lambda^{m}}{1-\lambda}\right) \geq \frac{4}{\varepsilon}\left(\frac{M+\varepsilon+1}{2 \lambda-1}\right) .
$$

Let $S=\left\{2^{n} \psi \mid 0 \leq n<l+m\right\}$, and let $\delta$ be the smallest distance between two distinct points of $S$. Choose $j \geq N$ such that $0<\left|2^{j} \varphi-\psi\right| \leq 2^{-l-1} \delta$, and let $\omega=2^{j} \varphi$. It is vital that $\omega \neq \psi$, and here is where the fact that $\varphi$ is irrational is used. By the definition of $\psi$, there are infinitely many $j \geq N$ for which $2^{j} \varphi$ is within $2^{-l-1} \delta$ of $\psi$, but $2^{j} \varphi=\psi$ for at most one value of $j$, since otherwise $\varphi$ would be eventually periodic (that is, rational). Now let $\tilde{f}$ be a $C^{1}$ function which satisfies the following conditions:

(i) $\tilde{f}(\theta) \geq 0$ for all $\theta \in T$;

(ii) $\tilde{f}(\theta)=0$ when $\left|\theta-2^{l} \omega\right| \geq 2^{l}|\omega-\psi|$;

(iii) $\tilde{f}\left(2^{l} \omega\right)=2^{l-1}|\omega-\psi| \varepsilon$, and this is the maximum value of $\tilde{f}$;

(iv) $\left|\tilde{f}^{\prime}(\theta)\right| \leq \varepsilon$ for all $\theta \in T$.

Notice that $\tilde{f}\left(2^{l} \omega\right)$ is only required to be $\varepsilon / 2$ times the radius of the neighborhood of $2^{l} \omega$ on which it is supported, thus condition (iv) can be satisfied. Notice that the interval of support of $\tilde{f}$ has length $2^{l+1}|\omega-\psi| \leq \delta$, and $2^{l} \psi$ 
is one of its endpoints. Since $2^{l} \psi \in S$, by the definition of $\delta$ there are no points in $S$ at which $\tilde{f}>0$. That is, $\tilde{f}\left(2^{n} \psi\right)=0$ for $0 \leq n<l+m$, and thus

$$
\begin{aligned}
W \tilde{f}(\psi) & =\sum_{n=0}^{\infty} \lambda^{n} \tilde{f}\left(2^{n} \psi\right)=\sum_{n=l+m}^{\infty} \lambda^{n} \tilde{f}\left(2^{n} \psi\right) \\
& \leq \sum_{n=l+m}^{\infty} \lambda^{n} 2^{l-1}|\omega-\psi| \varepsilon=\frac{\lambda^{l+m}}{1-\lambda} 2^{l-1}|\omega-\psi| \varepsilon \\
& =(2 \lambda)^{l} \frac{\lambda^{m}}{1-\lambda}|\omega-\psi| \frac{\varepsilon}{2} .
\end{aligned}
$$

On the other hand,

$$
\begin{aligned}
W \tilde{f}(\omega) & =\sum_{n=0}^{\infty} \lambda^{n} \tilde{f}\left(2^{n} \omega\right) \geq \lambda^{l} \tilde{f}\left(2^{l} \omega\right) \\
& =\lambda^{l} 2^{l-1}|\omega-\psi| \varepsilon=(2 \lambda)^{l}|\omega-\psi| \frac{\varepsilon}{2}
\end{aligned}
$$

Then by (3.3),

$$
\begin{aligned}
W \tilde{f}(\omega)-W \tilde{f}(\psi) & \geq(2 \lambda)^{l}\left(1-\frac{\lambda^{m}}{1-\lambda}\right)|\omega-\psi| \frac{\varepsilon}{2} \\
& \geq \frac{4}{\varepsilon}\left(\frac{M+\varepsilon+1}{2 \lambda-1}\right)|\omega-\psi| \frac{\varepsilon}{2}=2\left(\frac{M+\varepsilon+1}{2 \lambda-1}\right)|\omega-\psi| .
\end{aligned}
$$

Next since $\omega=2^{j} \varphi$ and $j \geq N$, by (3.2)

$$
W f(\psi)-W f(\omega) \leq \frac{M+1}{2 \lambda-1}|\omega-\psi| .
$$

Therefore

$$
\begin{aligned}
W(f+\tilde{f})(\omega)-W(f+\tilde{f})(\psi) & =W f(\omega)-W f(\psi)+W \tilde{f}(\omega)-W \tilde{f}(\psi) \\
& \geq-\frac{M+1}{2 \lambda-1}|\omega-\psi|+2\left(\frac{M+\varepsilon+1}{2 \lambda-1}\right)|\omega-\psi| \\
& >\frac{M+\varepsilon+1}{2 \lambda-1}|\omega-\psi| .
\end{aligned}
$$

We have established (3.1), which completes the proof.

In the following theorem, we prove that the opposite is true of $S(P)$ when $P$ is a periodic orbit.

\subsection{Theorem. If $P$ is a periodic orbit, then $S(P)$ does have an interior.}

Proof. We begin with an estimate which shows that $W$ is a bounded linear operator from $C^{1}(T)$ to the Hölder space $C^{\alpha}(T)$, where we define $\alpha=$ $-\log \lambda / \log 2$. Choose $f \in C^{1}(T)$, and let $M$ be the maximum value of $\left|f^{\prime}\right|$. 
Pick $\theta, \varphi \in T$, and let $m$ be the positive integer for which $2^{-m-1}<|\theta-\varphi| \leq$ $2^{-m}$. Then since the maximum distance between any two points in $T$ is $1 / 2$,

$$
\begin{aligned}
|W f(\theta)-W f(\varphi)| & =\left|\sum_{n=0}^{\infty} \lambda^{n}\left(f\left(2^{n} \theta\right)-f\left(2^{n} \varphi\right)\right)\right| \\
& \leq \sum_{n=0}^{\infty} \lambda^{n}\left|f\left(2^{n} \theta\right)-f\left(2^{n} \varphi\right)\right| \leq \sum_{n=0}^{\infty} \lambda^{n} M\left|2^{n} \theta-2^{n} \varphi\right| \\
& \leq M\left(\sum_{n=0}^{m-1}(2 \lambda)^{n}|\theta-\varphi|+\sum_{n=m}^{\infty} \lambda^{n} \frac{1}{2}\right) \\
& \leq M\left(\frac{(2 \lambda)^{m}-1}{2 \lambda-1} 2^{-m}+\frac{\lambda^{m}}{2(1-\lambda)}\right) \\
& \leq M\left(\frac{1}{2 \lambda-1}+\frac{1}{2-2 \lambda}\right) \lambda^{m} \\
& =\frac{M}{(2 \lambda-1)(2-2 \lambda)} \lambda^{-1}\left(2^{-m-1}\right)^{\alpha} \\
& \leq \frac{M}{\lambda(2 \lambda-1)(2-2 \lambda)}|\theta-\varphi|^{\alpha} .
\end{aligned}
$$

Now given a periodic orbit $P$, let $\delta$ be a third of the smallest distance between any two points of $P$. Let $f$ be any $C^{1}$ function which satisfies the following conditions:

(i) $0 \leq f(\theta) \leq 1$ for all $\theta \in T$;

(ii) $f(\theta)=0$ when $\min _{\varphi \in P}|\theta-\varphi| \geq \delta$;

(iii) $f(\varphi)=1$ for all $\varphi \in P$.

We claim that $f$ lies in the interior of $S(P)$. Since $f$ is maximized on the periodic orbit $P$, so is $W f$; we now estimate for $\varphi \in P$ how much $W f(\varphi)$ exceeds nearby values. For $\theta$ within $\delta$ of $\varphi$, let $m$ be the positive integer for which $2^{-m} \delta<|\theta-\varphi| \leq 2^{1-m} \delta$. Then since $\delta<\left|2^{m} \theta-2^{m} \varphi\right| \leq 2 \delta$ and $2^{m} \varphi \in P, f\left(2^{m} \theta\right)=0$, and thus

$$
\begin{aligned}
W f(\theta)-W f(\varphi) & =\sum_{n=0}^{\infty} \lambda^{n}\left(f\left(2^{n} \theta\right)-f\left(2^{n} \varphi\right)\right) \leq \lambda^{m}\left(f\left(2^{m} \theta\right)-f\left(2^{m} \varphi\right)\right) \\
& =-\lambda^{m}=-\lambda \delta^{-\alpha}\left(2^{1-m} \delta\right)^{\alpha} \leq-\lambda \delta^{-\alpha}|\theta-\varphi|^{\alpha} .
\end{aligned}
$$

Now if $\tilde{f} \in C^{1}(T)$ has norm less than $\lambda^{2}(2 \lambda-1)(2-2 \lambda) \delta^{-\alpha}$, by our earlier estimate

$$
W \tilde{f}(\theta)-W \tilde{f}(\varphi) \leq \frac{\lambda^{2}(2 \lambda-1)(2-2 \lambda) \delta^{-\alpha}}{\lambda(2 \lambda-1)(2-2 \lambda)}|\theta-\varphi|^{\alpha}=\lambda \delta^{-\alpha}|\theta-\varphi|^{\alpha},
$$

and therefore $W(f+\tilde{f})(\theta)-W(f+\tilde{f})(\varphi) \leq 0$ when $\theta$ is within $\delta$ of $\varphi$. Then $\varphi$ is on the facade of $W(f+\tilde{f})$, and by Proposition 22 so is the rest of $P$. We have thus shown that the open ball of radius $\lambda^{2}(2 \lambda-1)(2-2 \lambda) \delta^{-\alpha}$ centered at $f$ is contained in $S(P)$, so $f$ is in the interior of $S(\dot{r})$ as claimed. 
We remark that it is possible, given additional constraints on $f$, to prove that for $\tilde{f} \in C^{1}(T)$ sufficiently small, the facade of $W(f+\tilde{f})$ consists only of $P$ and its preimages. The argument is much like the proof of Proposition 2.1, except that the analogue of $(2.3)$ can be proven by a direct estimate.

\section{REFERENCES}

1. O. Decroly and A. Goldbeter, Multiple periodic regimes and final state sensitivity in a biochemical system, Phys. Lett. A 105 (1984), 196-200.

2. C. Grebogi, E. Ott, and J. A. Yorke, Basin boundary metamorphoses: changes in accessible boundary orbits, Phys. D 24 (1987), 243-262.

3. _ Chaotic attractors in crisis, Phys. Rev. Lett. 48 (1982), 1507-1510.

4. _ Crises, sudden changes in chaotic attractors, and transient chaos, Phys. D 7 (1983), 181-200.

5. _- Critical exponent of chaotic transients in nonlinear dynamical systems, Phys. Rev. Lett. 57 (1986), 1284-1287.

6. __ Fractal basin boundaries, long-lived chaotic transients, and unstable-unstable pair bifurcation, Phys. Rev. Lett. 50 (1983), 935-938.

7. E. G. Gwinn and R. M. Westervelt, Intermittent chaos and low frequency noise in the driven damped pendulum, Phys. Rev. Lett. 54 (1985), 1613-1616.

8. G. H. Hardy, Weierstrass's non-differentiable function, Trans. Amer. Math. Soc. 17 (1916), 301-325.

9. R. G. Holt and I. B. Schwartz, Newton's method as a dynamical system: global convergence and predictability, Phys. Lett. A 105 (1984), 327-333.

10. J. L. Kaplan, J. Mallet-Paret, and J. A. Yorke, The Lyapunov dimension of a nowhere differentiable attracting torus, Ergodic Theory Dynamical Systems 4 (1984), 261-281.

11. S. W. McDonald, C. Grebogi, E. Ott, and J. A. Yorke, Fractal basin boundaries, Phys. D 17 (1985), 125-153.

12. __ Structure and crises of fractal basin boundaries, Phys. Lett. A 107 (1985), 51-54.

13. F. C. Moon and G.-X. Li, Fractal basin boundaries and homoclinic orbits for periodic motion in a two-well potential, Phys. Rev. Lett. 55 (1985), 1439-1442.

14. I. B. Schwartz, Bistability, basins of attraction and predictability in a forced mass-reaction mode, Phys. Lett. A 106 (1984), 339-342.

15. S. Takesue and K. Kaneko, Fractal basin structure, Prog. Theoret. Phys. 71 (1984), 35-49.

16. Y. Yamaguchi and N. Mishima, Fractal basin boundary of a two-dimensional cubic map, Phys. Lett. A 105 (1984), 259-262.

Department of Mathematics, Stanford University, Stanford, California 94305

Institute for Physical Science and Technology, University of Maryland, College Park, Maryland 20742 (Current address of J. A. Yorke)

Current address (B. R. Hunt): NSWC, Code R44, Silver Spring, Maryland 20903-5000 